\title{
Research Article \\ External Ventricular Drain Infections: Risk Factors and Outcome
}

\author{
S. Hagel, ${ }^{1,2,3}$ T. Bruns, ${ }^{2,3}$ M. W. Pletz, ${ }^{1,2}$ C. Engel, ${ }^{4}$ R. Kalff, ${ }^{5}$ and C. Ewald ${ }^{5}$ \\ ${ }^{1}$ Center for Infectious Diseases and Infection Control, Jena University Hospital, Jena, Germany \\ ${ }^{2}$ Center for Sepsis Control \& Care (CSCC), Jena University Hospital, Jena, Germany \\ ${ }^{3}$ Department of Internal Medicine IV, Gastroenterology, Hepatology and Infectious Diseases, Jena University Hospital, Jena, Germany \\ ${ }^{4}$ Institute of Medical Informatics, Statistics and Epidemiology, University of Leipzig, Leipzig, Germany \\ ${ }^{5}$ Department of Neurosurgery, Jena University Hospital, Jena, Germany
}

Correspondence should be addressed to S. Hagel; stefan.hagel@med.uni-jena.de

Received 28 May 2014; Revised 21 October 2014; Accepted 1 November 2014; Published 17 November 2014

Academic Editor: Albert Eid

Copyright (C) 2014 S. Hagel et al. This is an open access article distributed under the Creative Commons Attribution License, which permits unrestricted use, distribution, and reproduction in any medium, provided the original work is properly cited.

\begin{abstract}
External ventricular drainage (EVD) is frequently used in neurosurgery to drain cerebrospinal fluid in patients with raised intracranial pressure. We performed a retrospective single center study in order to evaluate the incidence of EVD-related infections and to identify underlying risk factors. 246 EVDs were placed in 218 patients over a 30-month period. EVD was continued in median for 7 days (range 1-44). The cumulative incidence of EVD-related infections was 8.3\% (95\% CI, 5.3-12.7) with a device-associated infection rate of 10.4 per 1000 drainage days (95\% CI, 6.2-16.5). The pathogens most commonly identified were coagulase-negative Staphylococcus (62\%) followed by Enterococcus spp. (19\%). Patients with an EVD-related infection had a significantly longer ICU (11 versus 21 days, $P<0.01)$ and hospital stay $(20$ versus 28.5 days, $P<0.01)$ than patients without. Median total duration of external drainage was twice as long in patients with EVD-related infection ( 6 versus 12 days, $P<0.01$ ). However, there was no significant difference in the duration between first EVD placement and the occurrence of EVD-related infection and EVD removal in patients without EVD-related infection ( 6 versus 7 days, $P=0.87$ ), respectively. Interestingly no risk factor for EVD-related infection could be identified in our cohort of patients.
\end{abstract}

\section{Introduction}

Ventriculostomy catheters, also known as external ventricular drains (EVDs), are frequently used in neurosurgery to monitor and relief intracranial pressure. Complications arising from EVDs include hemorrhage, misplacement, dislodgement, blockage, and, most significantly, infection, which may be complicated by ventriculitis, meningitis, brain abscess, or subdural empyema. EVD-related infections significantly prolong hospital stay, increase costs, and often negatively affect the overall prognosis. Reported rates of EVD-related infections range from $<5 \%$ up to $23 \%$, most commonly close to $10 \%$ [1]. An increased risk of infection has been observed in patients with subarachnoid or intraventricular hemorrhage, in patients with concurrent systemic infections as well as with longer duration of catheterization, cerebrospinal (CSF) leakage, and frequent manipulation of the EVD system [2-4].
In addition we hypothesized that multibed accommodation of patients with EVD in a mixed surgical intensive care setting may constitute a risk factor for EVD-related infections. The objective of the present study was to assess the incidence and outcome of EVD-related infections and to identify new and confirm already known risk factors.

\section{Methods}

For this single-center retrospective study we included all patients over 18 years of age that underwent EVD placement during a 30-month period (January 2010 to June 2012) at our 1.500 bed tertiary center (Jena University Hospital, Germany). For drainage conventional (Promedics) and silverimpregnated catheter (VentriGuard) were used. All catheters were inserted under sterile conditions in the operating theatre using a tunneled procedure technique and a closed system 
for drainage. There was no policy of routine CSF sampling or replacement of catheters during the whole study period. The third-generation cephalosporin Ceftriaxone was given at $2 \mathrm{~g}$ once daily to all patients from insertion until EVD removal.

Patients that were not immediately transferred to the ICU after EVD insertion $(n=5)$, patients presenting with open skull fracture and CSF leakage $(n=4)$, and patients with active infection of the central nervous system (CNS) at first EVD implantation $(n=18)$ were excluded from the study. A total of 218 patients were included in the study. The 26-bed ICU is a mixed ICU treating patients of all surgical disciplines in four single-bed rooms, seven two-bed, and two four-bed rooms. Patients were followed up for 7 days after discharge from ICU. The charts of all patients were retrospectively reviewed and demographics, ASA-score (physical status classification of preoperative patients for anaesthetic risk assessment from the American Society of Anaesthesiologists), EVD-related data, type of accommodation, and underlying or arising healthcare-associated infections during ventricular drainage were documented. The study was approved by the institutional review board.

2.1. Definition of Infection. We defined EVD-related infection as (1) positive CSF culture result plus clinical symptoms or CNS pleocytosis/cell count increase, or (2) in the case of negative CSF culture, clinical symptoms, and CNS pleocytosis/cell count increase $[5,6]$. Healthcare-associated infections were defined using the CDC/NHSN surveillance definitions [7].

2.2. Statistical Analysis. Continuous values were expressed as median (range) and compared using the Mann-Whitney $U$ Test. Categorical data were presented as frequencies and percentages and compared using the chi-square test or Fisher's exact test where appropriate. Cox regression analysis and the Kaplan-Maier method were used to determine predictors of EVD-related infection. On the basis of Poisson distribution and Wilson-Score method, respectively, we calculated the confidence intervals for the incidence density and cumulative incidence. $P$ values $<0.05$ in two-sided testing were considered significant. All analyses were performed using IBM SPSS Statistics version 22 .

\section{Results}

Two hundred and forty-six EVDs were placed in 218 patients. External drainage was continued in median for 7 days (range $1-44)$ resulting in 1.725 catheter days (Table 1). The first and second EVD remained each in place for a median of 6 days (1st: range 1-19 days, 2nd: range 1-17 days), the third EVD for 12 days (range 9-16). One patient required a fourth and fifth EVD which remained in situ for 11 and 12 days, respectively. Indications for extraventricular drainage were nontraumatic subarachnoid and/or intraventricular hemorrhage in 133 (61\%) patients, traumatic brain injury with subarachnoid and/or intraventricular hemorrhage in 38 (17\%) patients, intracranial tumors in $25(12 \%)$ patients $(n=8$ benign; $n=17$ malignant), and others in $22(10 \%)$ patients $(n=16$ contusion/edema; $n=6$ hydrocephalus).
TABLE 1: EVD catheter data.

\begin{tabular}{lcc}
\hline EVD & Patients $(n)$ & Catheter days $(n)$ \\
\hline 1st EVD & 218 & 1515 \\
2nd EVD & 23 & 150 \\
3rd EVD & 3 & 37 \\
4th EVD & 1 & 11 \\
5th EVD & 1 & 12 \\
\hline
\end{tabular}

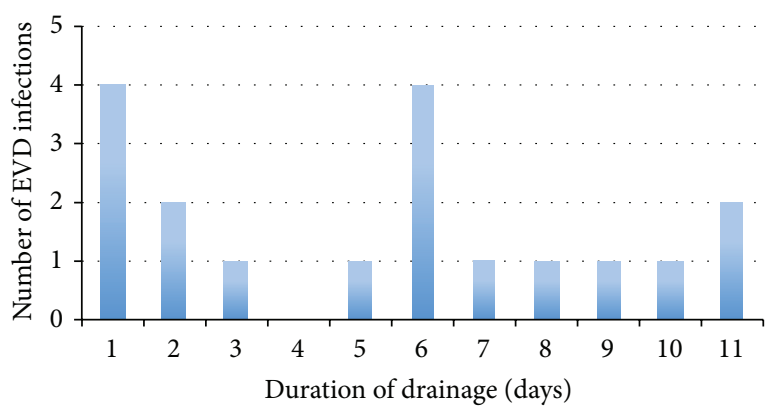

FIGURE 1: Occurrence of EVD-related infection related to drainage days after placement of EVD with underlying infection.

Eighteen patients developed an EVD-related infection resulting in a cumulative incidence of $8.3 \%$ (95\% CI, 5.312.7). All patients experienced only one episode of infection. In 14 patients the first EVD was infected, in four patients the second one. Six patients with an infection of the first EVD received a second EVD, one of those a third, and one patient five EVDs in total. Reasons for a change were the underlying infection and/or clogging with still necessity of ventricular drainage. Thirteen patients without an EVD-related infection received a second EVD; the reasons were displacement or clogging.

The device-associated infection rate was 10.4 per 1.000 (95\% CI, 6.2-16.5) EVD days considering the total time of EVDs in place (1.725 days) and 11.8 per 1000 (95\% CI, 6.9-18.6) EVD days considering catheter days without a previous EVD-related infection (1.572 days). On average an EVD-related infection became in mean evident 6 days (range 1-11) after insertion. Seven EVD-related infections were diagnosed within three days after EVD placement (Figure 1). In $88 \mathrm{EVD}$ procedures a conventional catheter was used and in 122 procedures a silver-impregnated catheter was inserted. In 26 procedures the type of the used catheter was not documented. No significant difference of EVD-related infection rate was found between patients with conventional EVD or silver-impregnated EVD (2\% versus $9 \% ; P=$ 0.08). Of 18 EVD infections, 16 (89\%) were proven based on microbiological test results. No polymicrobial infection was found. The pathogens most commonly identified were coagulase-negative Staphylococcus (CNS) (62\%) followed by Enterococcus spp. (19\%) and other pathogens (19\%) including Klebsiella pneumoniae, Escherichia coli, and Micrococcus luteus (Table 2).

No association of EVD-related infections with demographical parameters, indication for EVD placement, and 
TABLE 2: Microbial culture results of episodes of EVD infection.

\begin{tabular}{lc}
\hline Organism & $\begin{array}{c}n \text { (day of CSF sampling in } \\
\text { current EVD) }\end{array}$ \\
\hline $\begin{array}{l}\text { Enterococcus spp. } \\
\text { E. faecium } \\
\text { Unclassified }\end{array}$ & 2 (day 1,3$)$ \\
Klebsiella pneumoniae & 1 (day 6) \\
Staphylococcus spp. & 1 (day 5) \\
Staphylococcus hominis & 1 (day 10$)$ \\
Staphylococcus capitis & 1 (day 11$)$ \\
Staphylococcus haemolyticus & 1 (day 6) \\
Staphylococcus epidermidis & 7 (day $1,1,2,2,6,8,11)$ \\
E. coli & 1 (day 7) \\
Micrococcus luteus & 1 (day 6) \\
\hline
\end{tabular}

the type of accommodation could be detected (Table 3). Furthermore, there was no difference in mortality (singlebed $14 \%$ versus multibed $18 \%, P=1.0$ ) or in the occurrence of healthcare-associated infections other than EVDrelated infection between patients accommodated in singlebed rooms and multiple-bed rooms, respectively (21\% versus $25 \%, P=1.0)$. Concomitant healthcare-associated infections (HAIs) were found significantly more often in patients with EVD-related infections (44\% versus 23\%, $P<0.01$ ), with a trend towards more surgical site infections (SSI), urinary tract infections (UTI), and central line-associated bloodstream infections (CLABSI) in patients with EVD-related infections. No coherence between the organisms responsible for HAIs and those responsible for the EVD-related infection was found. Patients with an EVD-related infection had a significantly longer ICU (11 versus 21 days, $P<0.01$ ) and hospital stay (20 versus 28.5 days, $P<0.01$ ) than patients without. Median total duration of external drainage was twice as long in patients with EVD-related infection (6 versus 12 days, $P<0.01)$. However, there was no significant difference in the duration between first EVD placement and the occurrence of EVD-related infection, respectively, EVD removal in patients without EVD-related infection (6 versus 7 days, $P=0.87$ ). When controlled for the duration of the infection-free EVD drainage as a continuous variable, the occurrence of any HAI remained a statistically significant risk factor for the development of an EVD-related infection in a multivariate logistic regression model (univariate OR 4.3, 95\% CI 1.6-11.6; adjusted OR 7.1, 95\% CI 2.2-22.9), whereas the duration of drainage until infection or removal was not. Cox regression analysis did not identify a significant association between any further studied parameter and EVDrelated infection. A change of an EVD before infection or removal of the EVD was also not associated with an increased risk for a subsequent EVD-related infection $(P=0,47)$. EVD-related infection was not associated with increased inhospital mortality ( $17 \%$ versus $18 \% ; P=1.0)$ The only parameter associated with adverse outcome was ASA-score classification with an odds ratio of 2.7 per 1-class increase $(P<0.01)$.

\section{Discussion}

The cumulative incidence of EVD-related infection in our study was $8.3 \%$ and hence comparable with previous published studies [1-3]. The device-associated infection rate observed in this study (10.4 per 1000 EVD-days) is higher than the rate reported by Scheithauer et al. (6.3 per 1000 device days), which has been the only device-associated infection rate reported until now [5]. Noticeably, the mean duration of drainage time in patients without EVD-related infection in that study was considerably longer compared to our cohort (11 versus 8 days). The majority of published series reported a statistically significant higher incidence of EVD-related infections in patients with subarachnoid and/or intraventricular hemorrhage when compared with patients with nonhemorrhagic pathologies [1, 4]. According to two previous studies $[6,7]$ we however could not confirm subarachnoid and/or intraventricular hemorrhage as an independent risk factor for EVD-related infection in logistic regression analysis. Reasons for that discrepancy are not clear. Several studies examined the relationship between concurrent systemic infections and EVD-related infection, showing that concurrent systemic infections are a risk factor for EVD-related infection [1]. These results could not be confirmed in our study, in which the presence of a healthcare-associated infection during external ventricular drainage was not a risk factor for EVD-related infection. However, patients with an EVD-associated infection had significantly more often concurrent healthcare-associated infections, which might be a consequence of the prolonged length of stay in the intensive care unit. As in previous studies there was no coherence between the organism responsible for the healthcare-associated infection and the organism responsible for the EVD-related infection in the individual patient [1].

Multiple studies examined the duration of catheterization as a risk factor for EVD-related infection. Interpretation of these studies is complicated because some investigators used cumulative infection rates, either uncorrected or censored by the use of life-table analysis, while others used daily infection rates. Even though there is some controversy regarding the actual daily infection rate, Lozier et al. [1] could show that the hazard rate varies over time, suggesting daily changing infection risks. Although the median total duration of external drainage was twice as long in patients with EVDrelated infection than in patients without infection, there was no difference between insertion of the first EVD and occurrence of EVD-related infection or EVD removal in patients without EVD-related infection. This underlines the fact that prolongation of total drainage time in patients with EVD infection is a result of EVD infection and not vice versa. In line with these findings seven EVD-related infections were diagnosed within three days after placement of the infected EVD. Four of the seven EVD-related early infections were caused by coagulase-negative Staphylococci (CNS) which may have arisen from initial inoculations, which develop in detectable infections after variable incubation periods of around five days as previously suggested [8]. This emphasizes the necessity of EVD placement under fastidious aseptic 
TABLE 3: Patient demographics and outcome of EVD-related infections.

\begin{tabular}{|c|c|c|c|}
\hline & No EVD infection $(n=200)$ & EVD infection $(n=18)$ & $P$ \\
\hline Sex, $n(\%)$ & & & 0.46 \\
\hline Male & $111(93 \%)$ & $8(7 \%)$ & \\
\hline Female & $89(90 \%)$ & $10(10 \%)$ & \\
\hline Age (years) & $61(18-86)$ & $51(18-81)$ & 0.13 \\
\hline BMI & $25.5(18-45)$ & $25.5(18-48)$ & 0.65 \\
\hline ASA-score, $n(\%)$ & & & 0.46 \\
\hline 1 & $7(4 \%)$ & $0(0 \%)$ & \\
\hline 2 & $40(20 \%)$ & $2(11 \%)$ & \\
\hline 3 & $87(43 \%)$ & $11(61 \%)$ & \\
\hline 4 & $66(33 \%)$ & $5(28 \%)$ & \\
\hline Admission, diagnosis, $n(\%)$ & & & 0.54 \\
\hline $\mathrm{SAH}$, nontraumatic & $120(60 \%)$ & $13(71 \%)$ & \\
\hline $\mathrm{SAH}$, traumatic & $36(18 \%)$ & $2(11 \%)$ & \\
\hline Tumour, malignant & $17(8 \%)$ & $0(0 \%)$ & \\
\hline Tumour, benign & $7(4 \%)$ & $1(6 \%)$ & \\
\hline Hydrocephalus & $5(2 \%)$ & $1(6 \%)$ & \\
\hline Contusion/oedema & $15(8 \%)$ & $1(6 \%)$ & \\
\hline \multicolumn{4}{|l|}{ History, $n(\%)$} \\
\hline Cancer, haematology & $6(3 \%)$ & $0(0 \%)$ & 0.45 \\
\hline Cancer, solid & $18(9 \%)$ & $1(6 \%)$ & 0.62 \\
\hline Diabetes mellitus & $31(16 \%)$ & $1(6 \%)$ & 0.25 \\
\hline Immunosuppressive & $2(1 \%)$ & $0(0 \%)$ & 0.67 \\
\hline Neurosurgical procedure (besides of EVD placement) & $42(21 \%)$ & $3(17 \%)$ & 1.0 \\
\hline EVD-procedure, $n(\%)$ & & & 0.91 \\
\hline Elective & $24(12 \%)$ & $2(11 \%)$ & \\
\hline Emergency & $176(88 \%)$ & $16(89 \%)$ & \\
\hline Accommodation, $n(\%)$ & & & 0.50 \\
\hline Single-bed room & $12(6 \%)$ & $2(11 \%)$ & \\
\hline Double-bed room & $93(46 \%)$ & $7(39 \%)$ & \\
\hline Four-bed room & $95(48 \%)$ & $9(50 \%)$ & \\
\hline \multicolumn{4}{|l|}{ Concomitant infection, $n(\%)$} \\
\hline ANY & $45(23 \%)$ & $8(44 \%)$ & $<0.01$ \\
\hline LRTI & $42(21 \%)$ & $6(33 \%)$ & 0.23 \\
\hline SSI & $1(1 \%)$ & $2(11 \%)$ & 0.02 \\
\hline UTI & $0(0 \%)$ & $1(6 \%)$ & 0.08 \\
\hline CLABSI & $2(1 \%)$ & $2(11 \%)$ & 0.05 \\
\hline Other & $1(1 \%)$ & $0(0 \%)$ & 1.0 \\
\hline In-hospital death, $n(\%)$ & $36(18 \%)$ & $3(17 \%)$ & 1.0 \\
\hline External drainage, (total duration), days & $6(1-20)$ & $12(4-44)$ & $<0.01$ \\
\hline External drainage, (total duration of infection-free drainage) & $6(1-20)$ & $7(1-16)$ & 0.87 \\
\hline LOS-hospital, days & $20(1-90)$ & $28.5(15-74)$ & $<0.01$ \\
\hline LOS-ICU, days & $11(0-76)$ & $21(10-50)$ & $<0.01$ \\
\hline
\end{tabular}

SAH: subarachnoid hemorrhage, ASA: American Society of Anesthesiologists, LRTI: lower respiratory tract infection, SSI: surgical site infection, UTI: urinary tract infection, CLABSI: central line- associated bloodstream infection, LOS: length of stay.

conditions. In accordance with previous studies, coagulasenegative Staphylococci were the bacteria most commonly isolated in patients with EVD-related infections accounting for $62 \%$ of cases. Other common organisms include Enterococcus spp., Enterobacter spp., and Staphylococcus aureus [9]. This pattern coincides with that of the usual skin flora and hospital environment. Concerning our additional research question, whether multibed accommodation of patients in the ICU setting poses a risk factor for EVD-related infections we were not able to show any difference in the infection rate 
between patients who were placed in single- or multibed rooms, respectively.

The current notion is that EVD-related infections result from either inoculation of pathogens during EVD placement and/or contamination and colonization of the EVD system during the postoperative period [1]. Postoperative colonization can either arise from endogenous organisms present on the skin, which spread along the intracutaneous tract or by exogenous organisms introduced into the EVD system during manipulation at the EVD system by healthcare workers. Endogenous infections might be prevented by using antimicrobial coated EVD catheters which may decrease bacterial colonization and thus prevent infection. Just recently Wang et al. [10] performed a meta-analysis to assess the efficacy of antimicrobial-impregnated catheters in preventing catheter-related infections during external ventricular drainage. Compared with standard catheters, a significantly lower rate of CSF infection was noticed for clindamycin/rifampin-impregnated catheters (OR 0.27, 95\% CI, $0.10-0.73, P<0.05)$ and for minocycline/rifampinimpregnated catheters (OR 0.11, 95\% CI, 0.06-0.21, $P<0.05$ ). No statistical significance was found when standard catheters were compared with silver-impregnated catheters (OR 0.33, $95 \% \mathrm{CI}=0.07$ to $1.69, P=0.18)$. In the meantime however several additional studies were published which assessed the efficacy of silver-impregnated catheters. Winkler et al. [11] compared in their prospective randomized trial the rate of EVD-related infections of $61 \mathrm{EVD}$ placements with either antibiotic-coated $(n=32)$ or silver-bearing catheters $(n=29)$ in 40 patients. Regarding CSF infection rate and dysfunction, no statistical significant differences between the two EVD catheters Bactiseal versus VentriGuard were found. Lajcak et al. [12] performed a retrospective study of 403 patients with a total of 529 implanted EVDs. The rate of infections by catheter type was $7.6 \%(11 / 145)$ and $13.8 \%(4 / 29)$ for two different types of noncoated polyurethane catheters. Silverimpregnated polyurethane catheters became infected in $6.1 \%$ (14 out of 228). The differences between noncoated and silver-coated catheters were statistically significant. Keong et al. [13] performed a randomized controlled trail in overall 278 patients. There was a significant difference in infection risk between the two study arms: $21.4 \%$ (30/140) for plain catheters versus $12.3 \%(17 / 138)$ for silver catheters $(P=$ $0.04)$. In contrast to these studies, however, we could show a trend towards a higher infection rate in patients with a silver-coated EVD catheter when compared to patients with nonimpregnated catheters (9\% versus $2.3 \% ; P=0.08$ ). The reason for this observation is not obvious. Selection bias can be excluded as the catheters were not available in our institution at the same time.

4.1. Study Limitations. Because of the retrospective nature of the present study, various limitations must be mentioned. Particularly, data collection is compromised by missing values. Furthermore our findings might be limited due to the number of patients with EVD-related infection which was smaller than infected and the small number of patients who were accommodated in single-bed rooms.

\section{Conclusion}

Many studies have been conducted to identify risk factors of EVD-related infections. However, none of these risk factors could be confirmed in our cohort of patients. Furthermore we could not show any difference in infection rates between patients who were placed in single- or multibed rooms, respectively.

\section{Conflict of Interests}

The authors declare that there is no conflict of interests regarding the publication of this paper.

\section{Acknowledgments}

This study was supported by the Jena Center of Sepsis Control and Care (CSCC), funded by the German Ministry of Education and Research (BMBF; Grant 01 EO 1002).

\section{References}

[1] A. P. Lozier, R. R. Sciacca, M. F. Romagnoli et al., "Ventriculostomy-related infections: a critical review of the literature," Neurosurgery, vol. 51, no. 1, pp. 170-182, 2002.

[2] E. F. Camacho, Í. Boszczowski, M. Basso et al., "Infection rate and risk factors associated with infections related to external ventricular drain," Infection, vol. 39, no. 1, pp. 47-51, 2011.

[3] J.-H. Kim, N. S. Desai, J. Ricci et al., "Factors contributing to ventriculostomy infection," World Neurosurgery, vol. 77, no. 1, pp. 135-140, 2012.

[4] C. G. Mayhall, N. H. Archer, V. A. Lamb et al., "Ventriculostomy-related infections. A positive epidemiologic study," The New England Journal of Medicine, vol. 310, no. 9, pp. 553559, 1984.

[5] S. Scheithauer, U. Bürgel, Y.-M. Ryang et al., "Prospective surveillance of drain associated meningitis/ventriculitis in a neurosurgery and neurological intensive care unit," Journal of Neurology, Neurosurgery and Psychiatry, vol. 80, no. 12, pp. 13811385, 2009.

[6] M. Schultz, K. Moore, and A. W. Foote, "Bacterial ventriculitis and duration of ventriculostomy catheter insertion," The Journal of Neuroscience Nursing, vol. 25, no. 3, pp. 158-164, 1993.

[7] S. Scheithauer, U. Bürgel, J. Bickenbach et al., "External ventricular and lumbar drainage-associated meningoventriculitis: prospective analysis of time-dependent infection rates and risk factor analysis," Infection, vol. 38, no. 3, pp. 205-209, 2010.

[8] C. H. Lo, D. Spelman, M. Bailey, D. J. Cooper, J. V. Rosenfeld, and J. E. Brecknell, "External ventricular drain infections are independent of drain duration: an argument against elective revision," Journal of Neurosurgery, vol. 106, no. 3, pp. 378-383, 2007.

[9] P. M. Rath, B. Schoch, M. Adamzik, E. Steinmann, J. Buer, and J. Steinmann, "Value of multiplex PCR using cerebrospinal fluid for the diagnosis of ventriculostomy-related meningitis in neurosurgery patients," Infection, pp. 1-7, 2014.

[10] X. Wang, Y. Dong, X.-Q. Qi, Y.-M. Li, C.-G. Huang, and L.J. Hou, "Clinical review: efficacy of antimicrobial-impregnated catheters in external ventricular drainage-a systematic review and meta-analysis," Critical Care, vol. 17, no. 4, article 234, 2013. 
[11] K. M. L. Winkler, C. M. Woernle, M. Seule, U. Held, R. L. Bernays, and E. Keller, "Antibiotic-impregnated versus silverbearing external ventricular drainage catheters: preliminary results in a randomized controlled trial," Neurocritical Care, vol. 18, no. 2, pp. 161-165, 2013.

[12] M. Lajcak, V. Heidecke, K. H. Haude, and N. G. Rainov, "Infection rates of external ventricular drains are reduced by the use of silver-impregnated catheters," Acta Neurochirurgica, vol. 155 , no. 5, pp. 875-881, 2013.

[13] N. C. H. Keong, D. O. Bulters, H. K. Richards et al., "The SILVER (silver impregnated line versus evd randomized trial): a double-blind, prospective, randomized, controlled trial of an intervention to reduce the rate of external ventricular drain infection," Neurosurgery, vol. 71, no. 2, pp. 394-404, 2012. 


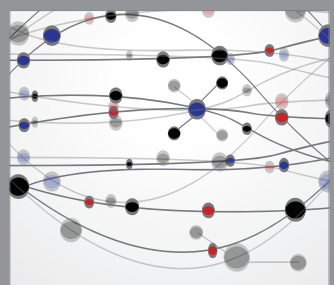

The Scientific World Journal
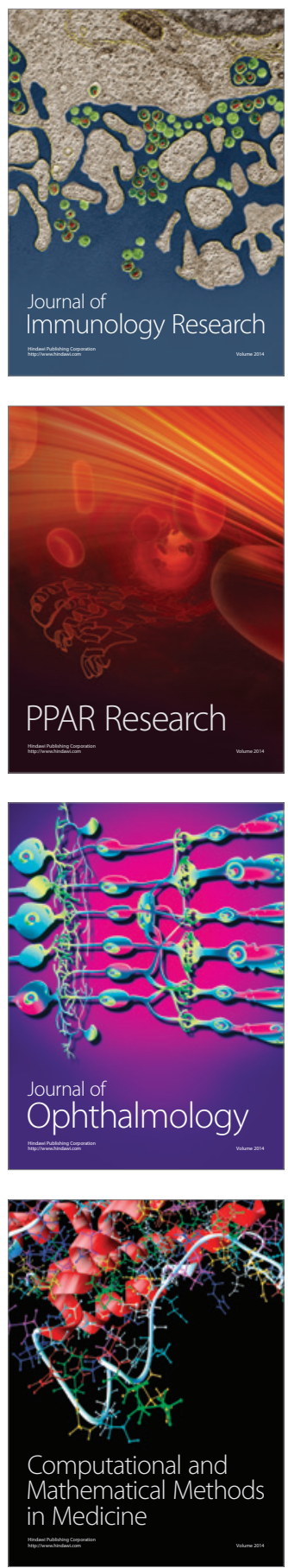

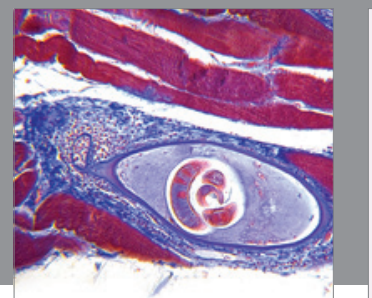

Gastroenterology

Research and Practice
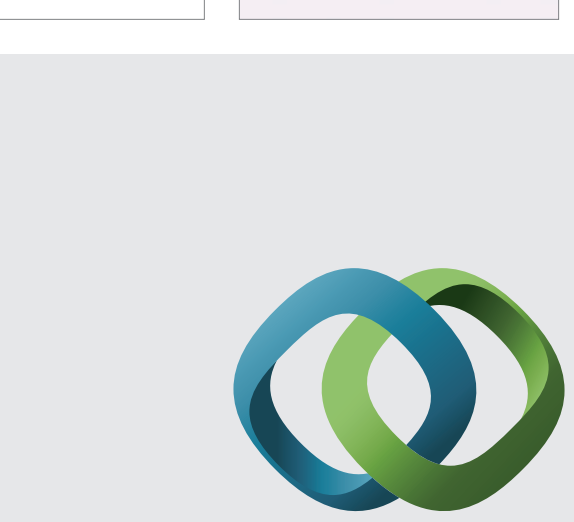

\section{Hindawi}

Submit your manuscripts at

http://www.hindawi.com
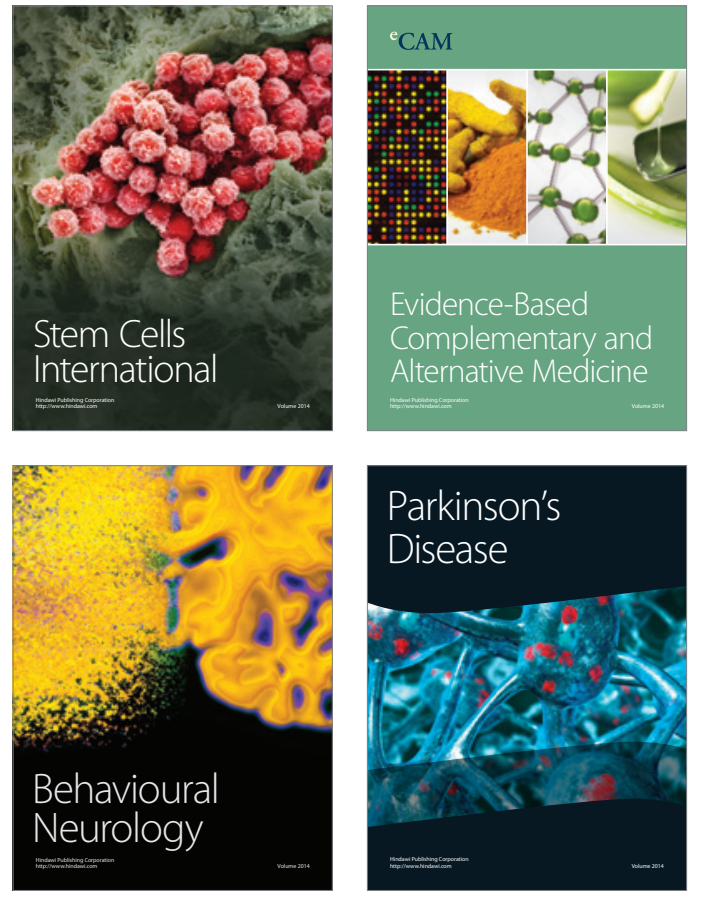
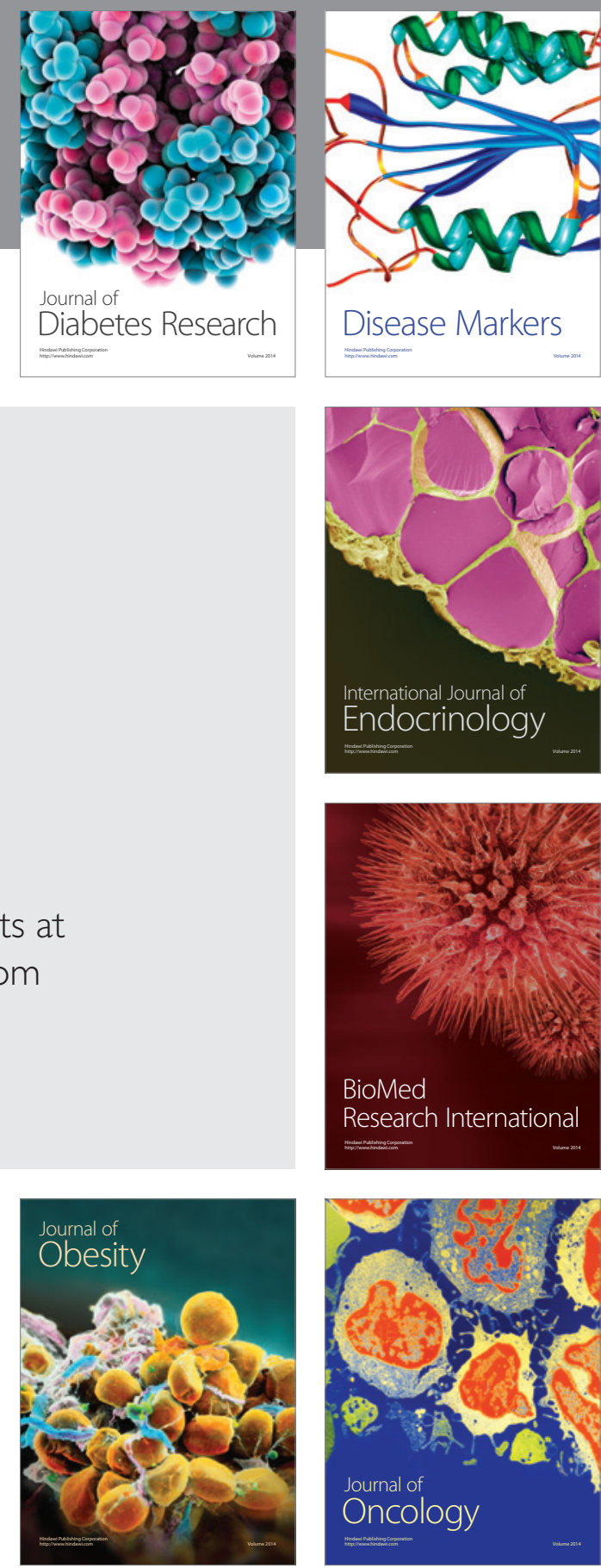

Disease Markers
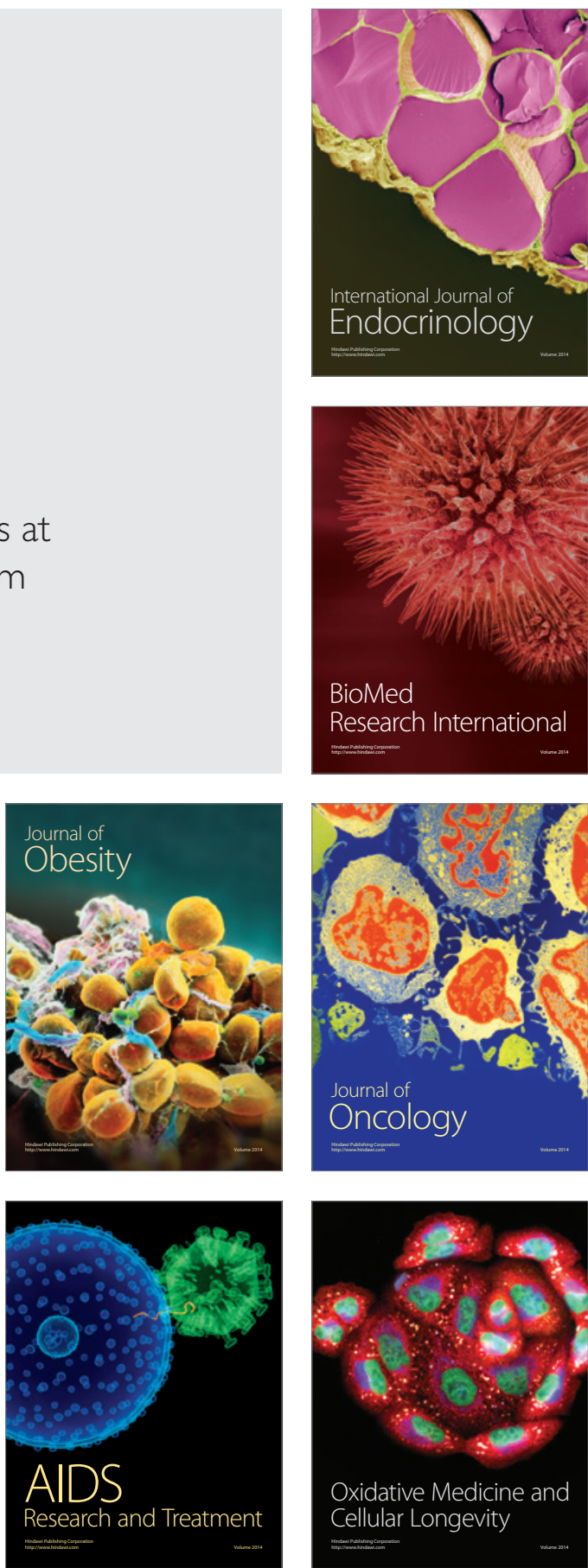